\title{
Triagem pré-participação em exercício físico em pacientes com doença arterial periférica
}

\author{
Exercise pre-participation screening in patients with peripheral arterial disease \\ Pollianny Ramos Lopes', João Paulo dos Anjos Souza Barbosa², Aluísio Henrique Rodrigues de Andrade Lima³, \\ Alessandra de Souza Miranda33, Lausanne Barreto de Carvalho Cahú Rodrigues², Sérgio Luiz Cahú Rodrigues4, \\ Raphael Mendes Ritti Dias ${ }^{5}$
}

\section{Resumo}

Contexto: Os indivíduos com doença arterial periférica (DAP) apresentam alto risco de doença cardiovascular. Dessa forma, é imprescindível que os instrumentos de triagem pré-participação em exercício indiquem quais pacientes com DAP devem realizar avaliação cardiovascular antes de iniciar o programa de exercício.

Objetivo: Comparar a sensibilidade de dois instrumentos de triagem pré-participação em exercício para identificação de indivíduos com DAP.

Métodos: Vinte e oito pacientes com DAP de ambos os sexos responderam ao questionário de prontidão para a atividade física (PAR-Q) e um questionário de estratificação do risco cardiovascular (QERC) baseado nas diretrizes do American College of Sports Medicine.

Resultados: O PAR-Q mostrou que a questão seis (Algum médico já recomendou o uso de medicamentos para a sua pressão arterial ou condição cardiovascular?) foi a que apresentou maior frequência de respostas positivas (82,1\%). Considerando todas as respostas, cinco indivíduos não tiveram nenhuma positiva ao questionário (17,9\%), indicando que estariam aptos para a prática de atividade física. Os resultados do QERC mostraram que as questões 11 (Você sente queimação ou sensação de câimbras em suas pernas quando faz caminhada?) e 2 (Algum médico já falou que você tem pressão alta?) apresentaram a maior frequência de respostas positivas (82,1\%).

Conclusões: Segundo o QERC, todos os indivíduos da amostra foram considerados com alto risco cardiovascular. O PAR-Q não foi sensível o suficiente para identificar todos os sujeitos, ao passo que o QERC identificou todos os indivíduos. Assim, sugere-se a utilização do QERC para identificação de indivíduos com DAP.

Palavras-chave: doença arterial periférica; exercício; saúde; avaliação; validade dos testes.

\begin{abstract}
Background: Exercise has been recommended as the main treatment for individuals with peripheral artery disease (PAD). However, since these individuals have increased cardiovascular risk, a cardiovascular assessment before onset of exercise program must be performed.

Objective: The purpose of this study was to compare the efficacy of two screening tools for identifying individuals with PAD.

Methods: Twenty-eight men and women with PAD, who answered the Physical Activity Readiness Questionnaire (PAR-Q) and the stratification of cardiovascular risk questionnaire (SCRQ).

Results: The PAR-Q results showed that question six (Has any physician recommended the use of blood pressure or cardiovascular medications?) had higher frequency of positive responses (82.1\%). Considering all responses, five individuals had all negative answers (17.9\%). The results of the SCRQ showed that the question 11 (Do you have leg cramps or burning sensation cramp when you walk?) and the $2^{\text {nd }}$ (Have you ever been told by a physician you have high blood pressure?) showed higher frequency of positive responses (82.1\%). According to the criteria for cardiovascular risk stratification, all individuals were considered to be at high cardiovascular risk.

Conclusion: The PAR-Q questionnaire was not sensitive enough to identify all subjects with PAD. On the other hand, the SRCQ was more efficient in identifying the subjects with PAD. Therefore, the use of SRCQ for screening PAD patients is suggested.
\end{abstract}

Keywords: peripheral arterial disease; exercise; health; evaluation; validity of tests.

\footnotetext{
Trabalho realizado na Escola Superior de Educação Física da Universidade de Pernambuco, Recife, PE, Brasil.

'Bacharel, Escola Superior de Educação Física, Universidade de Pernambuco, Recife, PE, Brasil

${ }^{2}$ Mestre, Escola Superior de Educação Física, Universidade de Pernambuco, Recife, PE, Brasil

${ }^{3}$ Mestrando, Escola Superior de Educação Física, Universidade de Pernambuco, Recife, PE, Brasil

${ }^{4}$ Mestre, Escola Superior de Educação Física, Universidade de Pernambuco, Recife, PE, Brasil

${ }^{5}$ Doutor, Escola Superior de Educação Física, Universidade de Pernambuco, Recife, PE, Brasil

Fonte de financiamento: Programa de Fortalecimento Acadêmico da Universidade de Pernambuco e Fundação de Amparo à Pesquisa do Estado de Pernambuco.

Conflito de interesses: nada a declarar.

Submetido em: 27.01.12. Aceito em: 12.03.12

J Vasc Bras. 2012;11(3):194-198.
} 


\section{Introdução}

A doença arterial periférica (DAP) refere-se à redução da luz das artérias que irrigam as regiões periféricas do corpo, causada por uma disfunção endotelial e consequente manifestação clínica da aterosclerose, promove desequilíbrio entre a demanda e oferta de oxigênio ${ }^{1,2}$. Estima-se que no Brasil a prevalência dessa doença seja de aproximadamente $10,5 \%$ na população acima dos 18 anos $^{3}$.

Indivíduos com DAP geralmente apresentam outras doenças cardiovasculares (DCV) associadas. Um estudo recente indicou que $93,3 \%$ dos pacientes com DAP têm hipertensão arterial sistêmica e $20 \%$ doenças cardíacas associadas ${ }^{4}$. Além disso, outras pesquisas demonstram que essa população apresenta grave prognóstico cardiovascular, ${ }^{5,6}$. De fato, indivíduos com DAP têm maior taxa de mortalidade quando comparados a pacientes sem DAP ou doenças cardiovasculares ${ }^{6}$.

Nesse sentido, programas sistematizados de exercício físico têm sido recomendados para essa populaçãa ${ }^{7,8}$. Eles promovem melhorias significativas na aptidão física ${ }^{9,10}$, qualidade de vida ${ }^{11}$ e função cardiovascular ${ }^{12-14}$. Além disso, os benefícios refletem em menor custo com internações hospitalares ${ }^{15}$. Entretanto, antes de iniciar programas de exercício físico sistematizado, esses indivíduos devem ser submetidos a uma triagem minuciosa, devido ao seu alto risco cardiovascular $^{16}$ e respostas cardiovasculares diferenciadas ao exercício $^{17-20}$.

Na prática das academias e dos centros de exercício físico, a indicação para a realização da avaliação cardiovascular é feita após a realização de uma triagem do risco cardiovascular, geralmente por meio de questionários. Para tanto, o questionário de prontidão para a atividade física (PAR-Q) $)^{21}$ é um dos instrumentos mais utilizados para essa finalidade. No entanto, recentemente, outros instrumentos também têm sido propostos, entre eles a estratificação do risco cardiovascular, de acordo com as diretrizes do American College of Sports Medicine $^{22}$. Os instrumentos apresentam como vantagens a praticidade, rapidez para a realização e uma sensibilidade relativamente alta (89\%) para identificação de indivíduos com doenças cardíacas ${ }^{21}$.

Apesar dessas vantagens, a capacidade dos questionários para identificação de indivíduos com DAP ainda não foi estabelecida. Esse é um aspecto importante, tendo em vista que a maioria dos instrumentos disponíveis na literatura para a estratificação do risco cardiovascular não inclui questões específicas referentes a essa doença, o que pode fazer com que o risco cardiovascular seja estratificado erroneamente. Dessa forma, o objetivo do presente estudo foi analisar e comparar a sensibilidade de dois instrumentos de triagem pré-participação para identificação de indivíduos com DAP.

\section{Métodos}

\section{Sujeitos}

A amostra foi composta por 28 indivíduos (17 homens e 11 mulheres) com idades entre 30 e 79 anos (62 \pm 12 anos). Após receberem esclarecimentos a respeito dos procedimentos do estudo, os indivíduos assinaram um Termo de Consentimento Livre e Esclarecido. A pesquisa foi conduzida dentro dos padrões éticos exigidos pela Declaração de Helsinque de 1964 e de acordo com a resolução 196/96 do Ministério da Saúde, tendo sido aprovada pelo Comitê de Ética da instituição dos autores.

Para inclusão no estudo, os indivíduos deveriam apresentar índice tornozelo braço (ITB) em repouso inferior a 0,90, referir sintomas de claudicação intermitente ou apresentar tolerância ao exercício limitada pelos sintomas de claudicação.

\section{Diagnóstico da doença arterial periférica}

O diagnóstico da DAP foi realizado por meio do ITB em repouso ${ }^{23}$. Para tanto, a pressão arterial sistólica do braço e do tornozelo nos dois membros foi medida em triplicata ${ }^{15}$. A aferição da pressão arterial no braço foi feita com o uso de um estetoscópio (Premium;, Brasil) e esfigmomanômetro de coluna de mercúrio (Unitec, Brasil). Para as medidas, o voluntário ficou em repouso, deitado por cinco a dez minutos. Para a medida da pressão arterial sistólica no tornozelo, foi utilizado um doppler vascular portátil (Martec DV 6000, Brasil) posicionado nas artérias pediosa e tibial posterior. Tanto para a medida no braço, quanto para a medida na perna, a pressão arterial sistólica foi identificada pelo aparecimento do primeiro som (fase I de Korotkoff). Em posse dos dados de pressão arterial do braço e de tornozelo, foi calculado o ITB por meio da divisão da maior pressão arterial sistólica do tornozelo pela maior pressão arterial sistólica do braço. Foram caracterizados como portadores da DAP aqueles que apresentaram ITB $<0,90$ em repouso em pelo menos um membro.

\section{PAR-Q}

O PAR-Q é composto por sete perguntas, entre as quais cinco estão relacionadas a sintomas cardiovasculares. Em cada questão o voluntário deveria relatar "sim" ou "não". De acordo com os idealizadores do instrumento ${ }^{21}$, o indivíduo 
que responder negativamente a todas as questões está apto a iniciar a prática do exercício físico.

\section{Estratificação de risco cardiovascular baseado nos critérios do American College of Sports Medicine}

Para estratificação do risco cardiovascular baseado nas diretrizes do American College of Sports Medicine ${ }^{22}$ foram obtidas informações sobre os fatores de riscos cardiovasculares, a presença de doenças cardíacas e a presença de sintomas durante a prática de exercício físico por meio de um questionário, denominado neste estudo de questionário de estratificação do risco cardiovascular (QERC). Todos os seus dados foram autorreferidos, exceto a obesidade, identificada pela relação cintura quadril maior que 0,95 para homens e 0,80 para mulheres e pelo índice de massa corporal $\geq 30,0 \mathrm{~kg} /$ $\mathrm{m}^{2}$. Para tanto, foram obtidas a circunferência da cintura e do quadril, por meio de uma fita antropométrica (Sanny, Brasil), a massa corporal, com uma balança de plataforma (Filizola, Brasil), e a estatura, por um estadiômetro de madeira. Em posse dos dados do questionário, os indivíduos foram estratificados em baixo, médio ou alto risco cardiovascular, de acordo com os critérios estabelecidos pelo American College of Sports Medicine ${ }^{22}$.

\section{Análise estatística}

Para análise dos dados, foi utilizada estatística descritiva com média e desvio-padrão e distribuição de frequências absolutas e relativas.

\section{Resultados}

Na Tabela 1 são apresentados os dados referentes às características dos sujeitos, as comorbidades e a escolaridade da amostra.

Já na Tabela 2 constam as frequências das respostas positivas em cada questão do PAR-Q. Os resultados mostraram que a questão seis foi a que apresentou maior frequência de respostas positivas $(82,1 \%)$, seguida das questões três $(28,6 \%)$ e um $(28,6 \%)$. Considerando todos os participantes avaliados, cinco indivíduos não tiveram nenhuma resposta positiva ao questionário (17,9\%).

Na Tabela 3 são demonstrados os dados referentes à frequência de respostas positivas em cada questão do QERC. Os resultados mostraram que as questões $11(82,1 \%)$ e $2(82,1 \%)$ apresentaram a maior frequência de respostas positivas, seguidas da 5 (53,6\%). De acordo com os critérios de estratificação do risco cardiovascular, todos os indivíduos da amostra foram considerados de alto risco.

\section{Discussão}

Os resultados deste estudo mostraram que alguns indivíduos com DAP foram considerados aptos para a prática de atividade física de acordo com o PAR-Q. Por outro lado, utilizando os critérios de estratificação do risco cardiovascular baseado no QERC, todos os indivíduos com DAP foram considerados com alto risco cardiovascular.

O PAR-Q foi proposto por Shephard et al. ${ }^{21}$, que verificou a sensibilidade e a especificidade desse instrumento em 1.130 sujeitos adultos. Os resultados indicaram alta sensibilidade, uma vez que todos os indivíduos com problemas de saúde diagnosticados pelo exame médico foram identificados pelo questionário. Em contrapartida, a especificidade observada nele foi baixa, apresentando que alguns sujeitos foram excluídos da prática do exercício físico desnecessariamente. Todavia, os resultados obtidos neste estudo indicaram que alguns dos indivíduos com DAP foram considerados aptos para a prática de atividade física. Os resultados corroboram com outra pesquisa realizada com 104 idosos de um programa de exercício físico supervisionado ${ }^{24}$, em que o mesmo observou que o PAR-Q, embora apresente sensibilidade alta (89\%), não permite identificar todos os indivíduos em risco, sugerindo que esse instrumento não seja adequado em populações específicas, o que descredenciaria o instrumento detectar casos verdadeiro-positivos.

Por outro lado, por meio dos dados obtidos no QERC verificou-se que todos os indivíduos com DAP apresentavam alto risco cardiovascular. Mais de $82 \%$ deles referiram sintomas de claudicação intermitente e, dentre aqueles que não referiram os sintomas, todos eram diabéticos. Essas duas características já inserem os indivíduos no estrato de alto risco. A melhor capacidade de identificação desse instrumento em comparação ao PAR-Q pode ser atribuída à questão específica sobre os sintomas de claudicação intermitente, que aumentou a capacidade do instrumento em identificar indivíduos com DAP. Além disso, no QERC são incluídas perguntas que

Tabela 1. Características dos indivíduos.

\begin{tabular}{ll}
\hline \multicolumn{1}{c}{ Variáveis } & \multicolumn{1}{c}{ Valores } \\
\hline Idade (anos) & $62,0 \pm 12,0$ \\
Massa corporal (kg) & $69,0 \pm 12,0$ \\
Estatura (m) & $1,60 \pm 0,10$ \\
Índice de Massa Corporal $\left(\mathrm{kg} / \mathrm{m}^{2}\right)$ & $27,5 \pm 3,5$ \\
Índice tornozelo braço & $0,71 \pm 0,14$ \\
\hline Fatores de risco & \\
\hline Hipertensão (\%) & 75,0 \\
Diabetes (\%) & 46,4 \\
Tabagismo (\%) & 25,0 \\
Cardiopatia (\%) & 35,7 \\
\hline
\end{tabular}


Tabela 2. Frequência de respostas positivas em cada questão do questionário de prontidão para a atividade física.

\begin{tabular}{|c|c|c|}
\hline Questões & $f(n)$ & $\%$ \\
\hline $\begin{array}{l}\text { 1. Alguma vez, um médico ou profissional de saúde disse que você possui problema no coração e recomendou que fizesse atividade física sob } \\
\text { supervisão médica? }\end{array}$ & 8 & 28,6 \\
\hline 2. Você sente ou já sentiu dor no peito quando faz atividade física? & 5 & 17,9 \\
\hline 3. Você sentiu dor no peito, sem fazer esforços, nos últimos meses? & 8 & 28,6 \\
\hline 4. Você tende a cair ou perder a consciência como resultado de tonteira? & 5 & 17,9 \\
\hline 5. Você tem algum problema ósseo, muscular ou articular que poderia ser agravado com a prática de atividade física? & 3 & 10,7 \\
\hline 6. Algum médico já recomendou o uso de medicamentos para a sua pressão arterial ou condição cardiovascular (ex: diuréticos e outros)? & 23 & 82,1 \\
\hline $\begin{array}{l}\text { 7. Você tem conhecimento, por meio de sua própria experiência ou aconselhamento médico, de alguma outra razão que o impeça de praticar } \\
\text { atividades físicas sem supervisão médica? }\end{array}$ & 3 & 10,7 \\
\hline Indivíduos que não tiveram pelo menos uma resposta positiva ao questionário: & 5 & 17,9 \\
\hline
\end{tabular}

Tabela 3. Frequência de respostas positivas em cada questão da estratificação do risco cardiovascular.

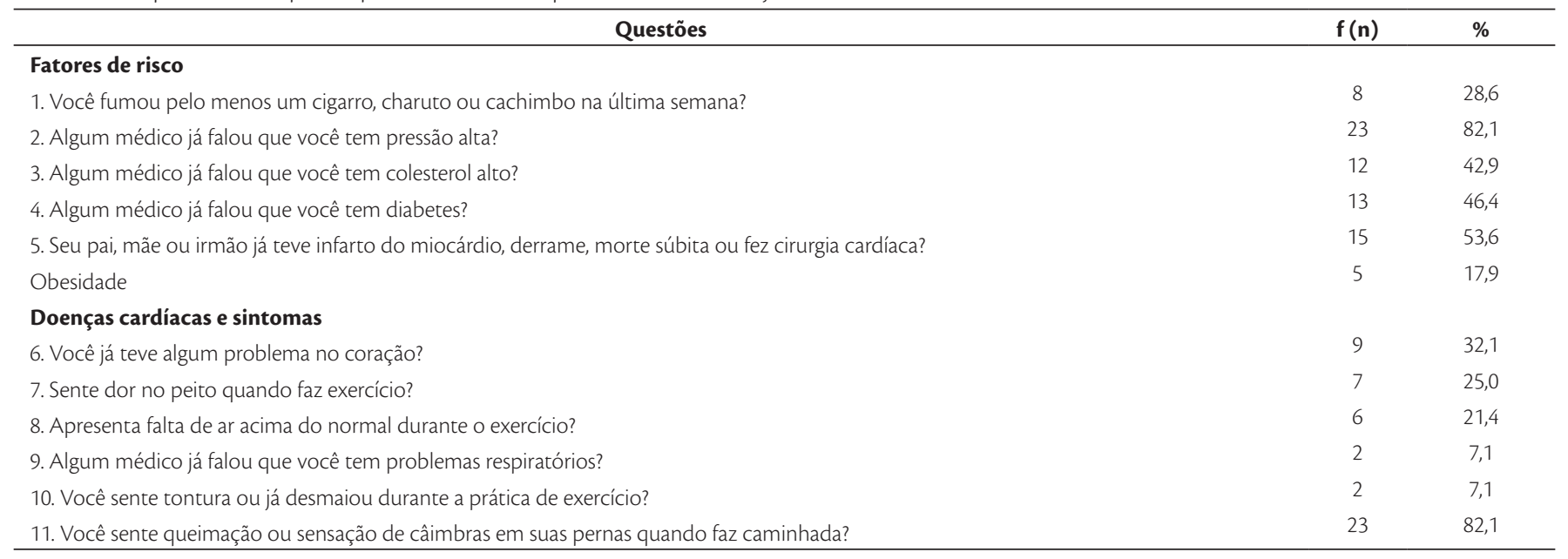

envolvem, além das DCV, as doenças metabólicas, importantes fatores de risco para as $\mathrm{DCV}^{25}$. No caso de indivíduos com DAP, por exemplo, o Diabetes Mellitus é um importante fator de risco para a doença, presente em mais de $30 \%$ dos indivíduos.

Em termos práticos, os resultados deste estudo indicam que o QERC parece ser um instrumento mais adequado para a identificação de indivíduos com DAP do que o PAR-Q. Dessa forma, a sua utilização pode ser uma alternativa interessante a ser empregada na prática no sentido de identificar o estado de prontidão de indivíduos para a realização de exercício físico, levando-a em consideração como recomendação inicial para esse tipo de população.

No entanto, a pesquisa apresenta algumas limitações que devem ser consideradas. A amostra, além de reduzida, foi composta por indivíduos com DAP que referiram sintomas de claudicação intermitente. Dessa forma, os dados do presente estudo não podem ser extrapolados para indivíduos com outros níveis de DAP. Além disso, os dados foram colhidos em amostra de hospitais, o que não pode representar a amostra de frequentadores de academias.
Como conclusão, os resultados obtidos indicam que o PAR-Q não foi sensível o suficiente para identificar todos os indivíduos com DAP. Em contrapartida, de acordo com o QERC, todos os indivíduos estavam com a doença. Assim, sugere-se a utilização do QERC para que seja possível a identificação de indivíduos com DAP em comparação ao PAR-Q.

\section{Referências}

1. Munger MA, Hawkins DW. Atherothrombosis: epidemiology, pathophysiology, and prevention. J Am Pharm Assoc. 2004;44(2 Suppl 1):S5-12. PMid:15095931.

2. Aragão JA, Reis FP, Borges Neto RR, et al. Prevalência da doença arterial obstrutiva periférica em doentes com insuficiência renal crônica. J Vasc Bras. 2009;8(4):301-6. http://dx.doi.org/10.1590/ S1677-54492009000400004

3. Makdisse M, Pereira AC, Brasil DP, et al. Prevalência e fatores de risco associados à doença arterial periférica no projeto corações do Brasil. Arq Bras Cardiol. 2008;91(6):402-14. http://dx.doi.org/10.1590/ S0066-782X2008001800008

4. Ritti-Dias RM, Wolosker N, De Moraes Forjaz CL, et al. Strength training increases walking tolerance in intermittent claudication 
patients: randomized trial. J Vasc Surg. 2010;51(1):89-95. http://dx.doi. org/10.1016/j.jvs.2009.07.118

5. Hirsch AT, Haskal ZJ, Hertzer NR, et al. ACC/AHA 2005 guidelines for the management of patients with peripheral arterial disease (lower extremity, renal, mesenteric, and abdominal aortic): executive summary a collaborative report from the American Association for Vascular Surgery/Society for Vascular Surgery, Society for Cardiovascular Angiography and Interventions, Society for Vascular Medicine and Biology, Society of Interventional Radiology, and the ACC/AHA Task Force on Practice Guidelines (Writing Committee to Develop Guidelines for the Management of Patients With Peripheral Arterial Disease) endorsed by the American Association of Cardiovascular and Pulmonary Rehabilitation; National Heart, Lung, and Blood Institute; Society for Vascular Nursing; TransAtlantic Inter-Society Consensus; and Vascular Disease Foundation. J Am Coll Cardiol. 2006;47(6):1239-312. PMid:16545667.

6. Pande RL, Perlstein TS, Beckman JA, Creager MA. Secondary prevention and mortality in peripheral artery disease: National Health and Nutrition Examination Study, 1999 to 2004. Circulation. 2011;124(1):17-23. PMid:3139992. http://dx.doi. org/10.1161/CIRCULATIONAHA.110.003954

7. Câmara LC, Santarém JM, Wolosker N, Dias RMR. Exercícios resistidos terapêuticos para indivíduos com doença arterial obstrutiva periférica: evidências para a prescrição. J Vasc Bras. 2007;6(3):246-56. http:// dx.doi.org/10.1590/S1677-54492007000300008

8. Norgren L, Hiatt WR, DormandyJA, etal. Inter-society consensus for the management of peripheral arterial disease. Int Angiol. 2007;26(2):81157. Pmid:17140820. http://dx.doi.org/10.1016/j.jvs.2006.12.037

9. Gardner AW, Katzel LI, Sorkin JD, Goldberg AP. Effects of long-term exercise rehabilitation on claudication distances in patients with peripheral arterial disease: a randomized controlled trial. J Cardiopulm Rehabil. 2002;22(3):192-8. PMid:12042688.

10. Watson L, Ellis B, Leng GC. Exercise for intermittent claudication. Cochrane Database Syst Rev. 2008;(4):CD000990. PMid:18843614.

11. Spronk S, Bosch JL, den Hoed PT, Veen HF, Pattynama PM, Hunink MG. Intermittent claudication: clinical effectiveness of endovascular revascularization versus supervised hospital-based exercise training-randomized controlled trial. Radiology. 2009;250(2):586-95. http:// dx.doi.org/10.1148/radiol.2501080607

12. Collins EG, Langbein WE, Orebaugh $C$, et al. Cardiovascular training effect associated with polestriding exercise in patients with peripheral arterial disease. J Cardiovasc Nurs. 2005;20(3):177-85. PMid:15870588.

13. Cucato GG, Ritti-Dias RM, Wolosker N, Santarem JM, Jacob Filho W, Forjaz CL. Post-resistance exercise hypotension in patients with intermittent claudication. Clinics. 2011;66(2):221-6. http://dx.doi. org/10.1590/S1807-59322011000200007

14. Grizzo Cucato G, De Moraes Forjaz CL, Kanegusuku H, et al. Effects of walking and strength training on resting and exercise cardiovascular responses in patients with intermittent claudication. Vasa. 2011;40(5):390-7. PMid:21948782.

15. Treesak C, Kasemsup V, Treat-Jacobson D, Nyman JA, Hirsch AT. Costeffectiveness of exercise training to improve claudication symptoms in patients with peripheral arterial disease. Vasc Med. 2004;9(4):27985. http://dx.doi.org/10.1191/1358863x04vm570oa

16. Yoshida RA, Matida CK, Sobreira ML, et al. Estudo comparativo da evolução e sobrevida de pacientes com claudicação intermitente, com ou sem limitação para exercícios, acompanhados em ambulatório específico. J Vasc Bras. 2008;7(2):112-22. http://dx.doi.org/10.1590/ S1677-54492008000200005
17. Dias RM, Forjaz CL, Cucato GG, et al. Obesity decreases time to claudication and delays post-exercise hemodynamic recovery in elderly peripheral arterial disease patients. Gerontology. 2009;55(1):21-6. PMid:18784409.

18. Gardner AW, Ritti-Dias RM, Stoner JA, Montgomery PS, Scott KJ, Blevins SM. Walking economy before and after the onset of claudication pain in patients with peripheral arterial disease. J Vasc Surg. 2010;51(3):628-33. http://dx.doi.org/10.1016/j.jvs.2009.09.053

19. Ritti-Dias RM, De Moraes Forjaz CL, Cucato GG, Costa LA, Wolosker N, de Fátima Nunes Marucci M. Pain threshold is achieved at intensity above anaerobic threshold in patients with intermittent claudication. J Cardiopulm Rehabil Prev. 2009;29(6):396-401. PMid:19770807.

20. Ritti-Dias RM, Meneses AL, Parker DE, Montgomery PS, Khurana A, Gardner AW. Cardiovascular responses to walking in patients with peripheral artery disease. Med Sci Sports Exerc. 2011;43(11):2017-23. PMid:21502888

21. Shephard RJ, Cox MH, Simper K. An analysis of "PAR-Q" responses in an office population. Can J Public Health. 1981;72(1):37-40. PMid:7225989.

22. American College of Sports Medicine - ACSM. ACSM's guidelines for exercise testing and prescription. Philadelphia: Lippincott Williams and Wilkins; 2006.

23. Wolosker N, Rosoky RA, Nakano L, Basyches M, Puech-Leão P. Predictive value of the ankle-brachial index in the evaluation of intermittent claudication. Rev Hosp Clin Fac Med Sao Paulo. 2000;55(2):614. http://dx.doi.org/10.1590/S0041-87812000000200005

24. Luz LGO, Maranhão Neto GA, Farinatti PTV. Validade do questionário de prontidão para a atividade física (PAR-Q) em idosos. Rev Bras Cineantropom Desempenho Hum. 2007;9(4):366-71.

25. Zerati AE, Wolosker N, Ayzin Rosoky RM, Fernandes Saes G, Ragazzo $\mathrm{L}$, Puech-Leão P. Prevalence of metabolic syndrome in patients with intermittent claudication and its correlation with the segment of arterial obstruction. Angiology. 2010;61(8):784-8. http://dx.doi. org/10.1177/0003319710366127

\section{Correspondência:}

Raphael Ritti-Dias

Escola Superior de Educação Física, Universidade de Pernambuco (UPE), Rua Arnóbio Marques, 310, Santo Amaro, CEP 50100-130, Recife, PE, Brasil E-mail: raphaelritti@gmail.com

Contribuições dos autores: Concepção e desenho do estudo: PRL, RMR. Análise e interpretação dos dados: PRL, SLCR, RMR. Coleta de dados: AHRAL, ASM, JPASB, LBCCR. Redação do artigo: PRL, AHRAL, JPASB. Revisão crítica do texto: SLCR, RMR. Aprovação final do artigo*: PRL, JPASB, AHRAL, ASM, LBCCR, SLCR, RMRD. Responsabilidade geral pelo estudo: RMR *Todos os autores leram e aprovaram a versão final submetida ao I Vasc Bras. 